\title{
The VisLab Intercontinental Autonomous Challenge: an extensive test for a platoon of intelligent vehicles
}

\author{
Alberto Broggi, Pietro Cerri, Mirko Felisa, \\ Maria Chiara Laghi, Luca Mazzei* and \\ Pier Paolo Porta
}

VisLab, Artificial Vision and Intelligent System Lab,

Department of Information Engineering,

University of Parma, 181/A, G.P. Usberti,

I-43124 Parma, Italy

E-mail: broggi@vislab.it

E-mail: cerri@vislab.it

E-mail: felisa@vislab.it

E-mail: laghi@vislab.it

E-mail: mazzei@vislab.it

E-mail: portap@vislab.it

${ }^{*}$ Corresponding author

\begin{abstract}
This paper presents the VisLab Intercontinental Autonomous Challenge (VIAC), an autonomous vehicles test carried out from Parma to Shanghai between July and October 2010 by the VisLab team. The vehicle equipment is explained introducing the sensing systems which were tested during the journey. Trip details and the first statistics are presented as well.
\end{abstract}

Keywords: vehicle autonomous systems; platooning; computer vision.

Reference to this paper should be made as follows: Broggi, A., Cerri, P., Felisa, M., Laghi, M.C., Mazzei, L. and Porta, P.P. (2012) 'The VisLab Intercontinental Autonomous Challenge: an extensive test for a platoon of intelligent vehicles', Int. J. Vehicle Autonomous Systems, Vol. 10, No. 3, pp.147-164.

Biographical notes: Alberto Broggi received the Eng. (Master) degree in Electronic Engineering and the $\mathrm{PhD}$ degree in Information Technology both from the Universita di Parma, Italy, in 1990 and 1994, respectively. He is now Full Professor at the Universita di Parma. He is the Director of the Artificial Vision and Intelligent Systems Lab, VisLab and author of more than 150 publications on International Scientific Journals, book chapters, refereed conference proceedings. He served as Editor-in-Chief of the IEEE Transactions on Intelligent Transportation Systems for the term 2004-2008; he is now serving the IEEE Intelligent Transportation Systems Society as President for the term 2010-2011.

Pietro Cerri received the Eng. degree in Computer Engineering from the Universita di Pavia, Pavia, Italy, in 2003. In 2007, he received the PhD degree in Information Technologies from the Universita di Parma, Parma, Italy. He works as Research Engineer at Vislab, a spinoff company of Parma University. His reasearches are mainly focused on computer vision and sensors fusion approaches for the development of advanced driver assistance systems. 
Mirko Felisa received the Master degree in Computer Engineering from the Universita di Parma, Parma, Italy, in 2005. In 2010, he received the PhD degree in Information Technologies. He works as Research Engineer at Vislab, a spinoff company of Parma University. His reasearches are mainly focused on stereovision algorithms for automotive applications.

Maria Chiara Laghi received the Master degree in Computer Engineering from the Universita di Parma, Italy, in 2004. In 2010, she received the PhD degree in Information Technologies from the same University. She works as Research Engineer at Vislab, a spinoff company of Parma University. Her research activity focuses on intelligent systems for traffic analysis.

Luca Mazzei received the Master degree in Computer Engineering from the Universitaa di Parma, Parma, Italy, in 2008. He is a PhD candidate at Dipartimento di Ingegneria dell'Informazione of Parma University. His reasearches are mainly focused on computer vision and artificial intelligence algorithms for automotive applications and video surveillance.

Pier Paolo Porta received the MSc degree in Computer Engineering and the $\mathrm{PhD}$ degree in Information Technology from the Universitaa di Parma, Italy, in 2005 and 2010, respectively. He works as Research Engineer at Vislab, a spinoff company of Parma University. His research activity field is on autonomous vehicle and driver assistance system and is focused on short range obstacle detection, camera calibration and traffic sign recognition.

\section{Introduction and motivation}

In the last few years many research groups have been focusing their efforts on unmanned systems and specifically on driverless vehicles. Apart from the early years when, in the 1990s, only a few Universities were researching on driverless cars and some efforts were put in place by the military sector to enhance their vehicles with autonomous features, in the XXI century a renovated interest in these topics bloomed around the world.

The DARPA Challenges (Ozguner et al., 2004; Braid et al., 2006a; Chen et al., 2008) were the most important events that pushed many research teams to build driverless vehicles and face real environments and real scenarios. In 2004, 2005 and 2007, DARPA organised races amongst autonomous vehicles with different rules: in unknown off-road scenarios and in urban settings with other traffic. Many teams fielded successful entries in these events, progressing the overall knowledge in this field.

After these events, the world crisis struck almost all countries and in 2009 very few research centres and companies had the strength to support highly risky research efforts like these. Nonetheless, some advances in unmanned vehicular driving were conceived, hoping to raise a sufficient interest to attract funds and implement these ideas. And VisLab's entrepreneurism was no exception: thanks to a ERC Advanced Grant, VisLab had the possibility to continue working on this subject and improve the architecture of the BRAiVE prototype vehicle.

BRAiVE, (Grisleri and Fedriga, 2010) is VisLab's main testing prototype; it includes a unique sensing suite composed of 10 cameras, 4 laserscanners, 16 laser beams, 1 radar, GPS and IMU. BRAiVE's preparation was started just after the DARPA Urban Challenge 
as a further step in capitalising all the knowledge developed by VisLab in the last 10+ years. The original idea was to port all the software ever developed onto the BRAiVE platform and test it. Plus, BRAiVE had to become the main autonomous vehicle on which VisLab would develop all the new functions. By fusing data coming from all sensors, BRAiVE was then turned into a fully autonomous car that was able to perceive vehicles, lanes, obstacles, pedestrians, traffic signs and other environmental characteristics such as road slope.

BRAiVE was then demonstrated to move autonomously in selected regions and for given scenarios. Although all demos were successful, the main question that still had to find an answer was whether those perception systems could really work in all scenarios. In other words, whether perception was robust with respect to adverse weather, day and night, chaotic traffic patterns, different road infrastructures and other situations that might not have been considered in the design stage.

VisLab, then, decided to undertake a huge and unique test of their perception systems to evaluate their behaviour in very different environments, with the final aim of locating the critical situations. A further analysis of these situations would have brought their perception systems to an operational level comparable with possible products.

Instead of using BRAiVE as a test vehicle, VisLab designed a new concept vehicle that had to be used just for this enormous test: its technology was taken from BRAiVE and its architecture borrowed from BRAiVE's design. The new vehicle had to mimic BRAiVE's behaviour in order to infer its possible critical issues.

More issues were then brought onboard this new concept vehicle: instead of gasoline, propulsion was provided by batteries; new $\mathrm{CO}_{2}$ sensors were also hosted on the vehicle for a different test; and specific driving patterns were designed for this vehicle. Being an electric engine, its autonomy was limited and VisLab conceived a way to improve it: four vehicles were equipped instead of just one and they were swapped on the fly to replace vehicles with exhausted batteries with vehicles with fresh batteries.

All data acquired by the sensors during the test were saved for further analysis; the final goal was to capture a huge amount of data to create a sufficiently large set of scenarios to test the system robustness and improve it once back in laboratory after the test.

The VisLab Intercontinental Autonomous Challenge (VIAC) was then born in this way, as a huge test, carried out during 3 months (between July 26 and October 26, 2010). The route followed started in Parma and ended in Shanghai, at the 2010 world Expo.

This paper presents this worldwide test and describes the main results obtained so far. Section 2 presents the logistics of the entire journey, section 3 explains the vehicles' selection and their equipment. Section 4 presents an overview of the perception systems and Section 5 shows dates, routes and results.

\section{The test logistics}

One of the project partners, Overland Network, took care about logistics. This was a primary issue, since the trip crossed several countries, most of them requiring specific documents and visas to enter the vehicles and operate them inside their borders. The four autonomous vehicles had special licence plates provided by the vehicle manufacturer, Piaggio, specifically for testing.

For all the crossed countries no specific permissions were necessary, except China, which required a temporary Chinese licence plate plus a temporary Chinese driver's licence for every driver (or "person behind the steering wheel"). Plus, upon entering China, the vehicles had to pass additional tests such as headlights and braking tests. 
The expedition (shown in Fig. 1) was composed of 11 vehicles: four autonomous vehicles, three trucks used as storage of spare parts and mechanical shop and four motorhomes used as accomodation for the team. Shifts were organised to keep about six members of the VisLab team in the expedition for the whole trip. People were exchanged in group of two so that the experience gained could be passed on from one team member to the next. The other individuals following the expedition were provided by Overland Network. They were in charge of driving the supporting trucks and motorhomes and providing mechanical help. A member of the VisLab team was always present on the the autonomous vehicle driver seat, during autonomous driving, according to traffic law requirements, ready to manage unexpected situations or eventual failures. However, during live demos, which took place in major cities and were held in delimited areas, the automatic system was shown with nobody on the driver seat.

Figure 1 The whole expedition stopped at the side of the road. After the two autonomous vehicle, four motorhomes, then two supporting trucks and a tow truck with the other two autonomous vehicles on board (see online version for colours)

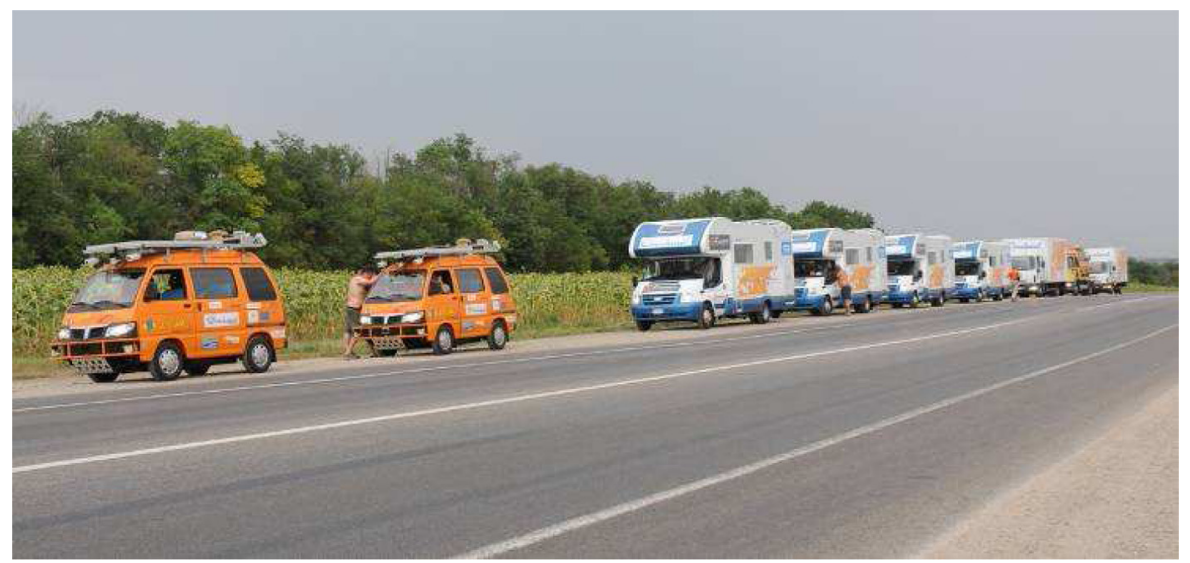

A diesel motor generator was located on the tow truck and used to recharge the vehicles batteries in remote locations where no power outlet was available.

Spare parts were loaded in the support trucks to perform maintenance and for mechanical repairs.

Calibration tools and instruments were also loaded in the support trucks to perform periodic calibration procedures related to all sensors. Calibration tasks are explain in section 4 .

\section{Vehicles and equipment selection}

\subsection{Vehicles selection}

Different vehicles were considered as candidates for this challenge. As there was no a-priori knowledge on the expected scenarios (urban, rural, off-road and highway environments could be encountered during the trip) the environment could not be used to drive the vehicles selection. Plus, since one of the key ideas that generated the idea of running this challenge was the vision for a future mobility that should be green, self-sustainable and safe, electric vehicles were chosen, as a challenge within the challenge. Thanks to the cooperation with Piaggio, the vehicles selected for VIAC were Piaggio Porter Electric Power. 
This choice was not easy because such a small vehicle seemed to be the less recommended vehicle for such a long, complex and extreme journey; on the other hand this was a good choice in view of a possible exploitation of this technology in urban applications as presented by van Dijke (2009). This vehicle was developed to fit these needs: it is small, agile and with no emissions.

Experiences about the use of electric vehicles for long trips were already carried out by team Venturi, as presented in Venturi (2010). Their second challenge was completed on July 13,2010 , just a few days before VIAC's official start: two and a half months on the road from Shanghai to Paris, with an electric car, but with drivers on it!

The VisLab group already gained experience on different autonomous vehicles, such as during its involvment in the DARPA Grand Challenges (cfr. Braid et al., 2006b) and the DARPA Urban Challenge (cfr. Chen et al., 2009). VisLab also equipped other intelligent vehicles such as ARGO, presented by Broggi et al. (1999) and, more recently, BRAiVE (Bombini et al., 2009), as presented in Section 1.

\subsection{Sensors selection}

Sensors' selection is a critical issue, because different policies can be considered. Price is a key factor: the use of expensive sensors, usually highly accurate, precise and with high resolution, generally simplifies the processing stage, but the risk is that the result will be too far away from real applications in the short term.

Redundancy can be guaranteed using a number of different sensors with overlapping fields of view, but the use of many sensors complicates the following fusion stage; sometimes this approach capitalises the capabilities of the different sensors, but in other cases it accumulates the problems of each single sensor, therefore requiring a very effective sensor fusion.

The chosen policy is a trade-off between all these different aspects.

The four electric vehicles were all equipped with the same sensing, processing and actuation technologies to optimise development time and usability. Nonetheless, the possibility of swapping the vehicles in operation (e.g., a leader, or a follower, or a standalone car) provides additional flexibility in case of failures or when there was the need for additional driving shifts due to the batteries' limited autonomy.

The sensor suite is based on the BRAiVE experience, except for an additional laser scanner, oriented to frame the ground, that is used primarily during off-road driving, which is not included in BRAiVE. However the integration level is completely different from what was decided for BRAiVE: in this case the sensors and all equipment had to be kept handy for easy maintenance even in remote locations and in extreme conditions (desert, mountain, remote areas).

Computer vision is widely used in this project. Seven cameras are installed on the vehicle (five forward and two backward looking), while four laser scanners with different characteristics are placed around the vehicle. Four cameras are mounted outside the cabin, attached to a roof rack that was added to support a number of sensors: this is the highest position allowed on the vehicle. The other three cameras are mounted inside the cabin, providing a panoramic view with an angle of about 180 degrees to simulate the driver's field of view. Redundancy is guaranteed in the area in front of the vehicle with four laser scanners and five cameras. A four-layer laser scanner is mounted in front of the vehicle, in the middle of the frontal bumper, to frame an area of about 100 degrees, while two other laser scanners are mounted on the front-right and front-left corners to frame the front and the sides of the vehicle. A bull bar is added to protect these laser scanners. The fourth laser scanner is mounted on the roof rack and tilted down to frame the ground. 
The vehicle also features GPS, IMU and V2V communication systems. A TopCon AGI3 inertial and geolocation device is installed on the vehicle roof. The GPS uses the Egnos/ WAAS correction in order to achieve about $1 \mathrm{~m}$ accuracy. The IMU is formed by two threeaxes gyroscopes, a three-axes accelerometer and a two-axes magnetometer. The TopCon system integrates also an ArWest AW401Tc radio-modem. This device works at 19200 Baud on an half-duplex digital communciation system and is able to transmit up to $24 \mathrm{~km}$ with a power of $1000 \mathrm{~mW}$ using an ominidirectional antenna. Figure 2 shows the sensors and devices installed on the vehicle.

Figure 2 Front: (a) and rear and (b) views of the vehicle (see online version for colours)

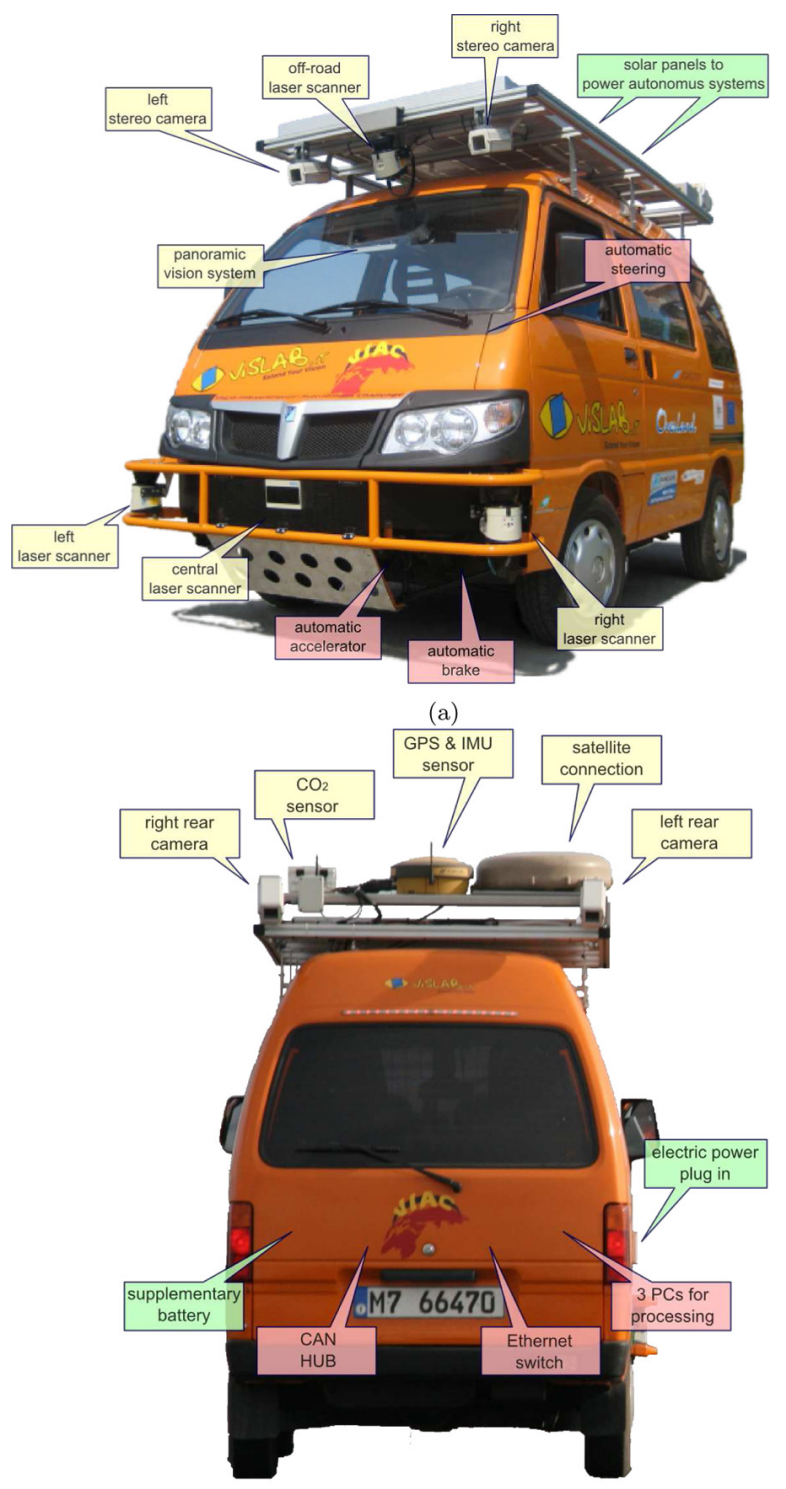

(b) 
Figure 3 Output of some of the systems installed on the vehicles: (a) lane detection; (b) preceding vehicle detection. Other algorithms, such as; (c) terrain mapping and (d) pedestrian detection, are being tested offline using the data collected during the expedition (see online version for colours)

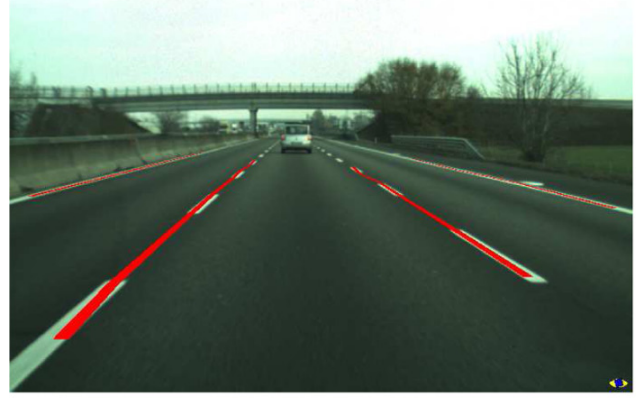

(a)

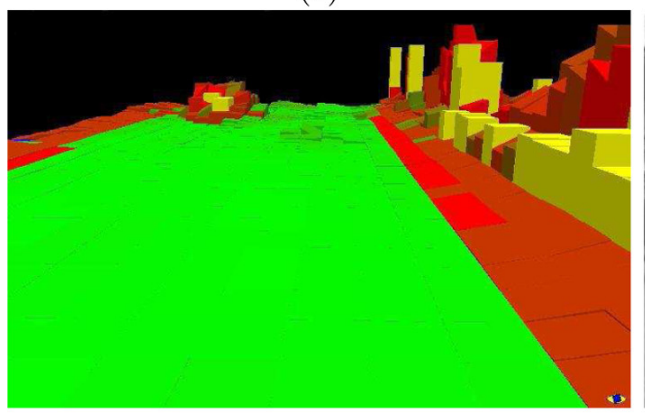

(c)

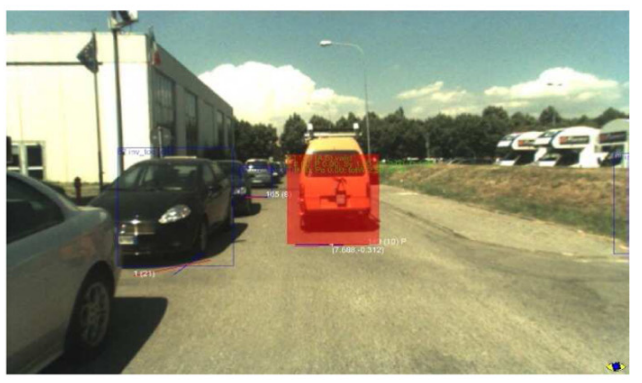

(b)

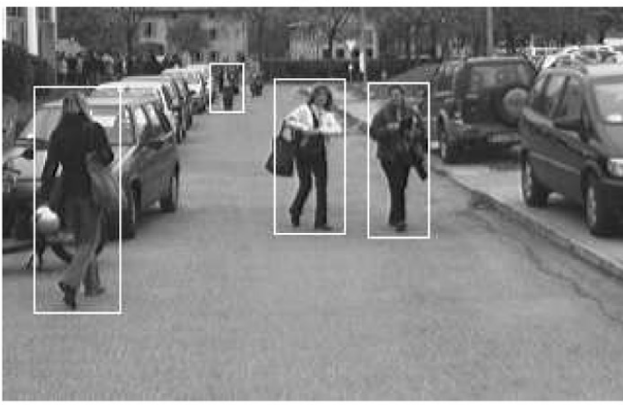

(d)

The onboard sensor suite is managed by three PCs, all featuring off-the-shelf boards and components. PC1 manages the GPS, IMU, x-by-wire and V2V communication systems; PC2, the three frontal cameras and the four laser scanners; finally PC3 manages the two stereo camera systems.

\subsection{Actuation devices}

The vehicle is equipped with full $\mathrm{x}$-by-wire, allowing to control speed and steering via CAN messages. Three specific systems have been designed to manage the steering, the brake and the engine throttle.

A servo motor, provided by TopCon, is directly connected to the steering wheel column; messages sent on a specific CAN-BUS can control position, speed and torque.

Speed is easily controlled by changing the duty cycle of the PWM that controls the electric engine. A specific board has been designed and developed which is able to select the gear (forward/backward), the working mode (automatic/manual) and the throttle value: when power is off the system defaults onto manual control. Instead of using a potentiometer, the value is discretely set using a number of relays which drive resistors with different values.

Finally, braking was realised by simply coupling an electric linear actuator to the braking pedal using a steel cable: by pulling the cable, braking is modulated. 


\subsection{Other devices}

Other devices have been installed to complement the sensing system and add further functions. A solar panel is used to recharge an additional car battery, which is used to power all sensors, processing systems and actuators.

An E-Stop function is available via RF signal to stop all autonomous vehicles at same time in dangerous situations.

A satellite communication equipment is installed on the vehicle roof to broadcast live info from the vehicle to the VisLab website. During the journey, the experiment was broadcast via live streaming thanks to a webcam installed on one of the vehicles. Live images were visible from the project web site.

$\mathrm{A} \mathrm{CO}_{2}$ sensor with GPS is also mounted on the vehicle for the Greenhaviour project, in collaboration with IBM (IBM, 2010).

\section{Systems under testing}

As mentioned in the previous section the VIAC was conceived to be a thorough test of all perception systems, to evaluate their behaviour in completely different environments and their stability after hours of continuous operation, with the final aim of locating possible critical situations. This section provides a list of the functions that were candidates for testing during the challenge.

\subsection{Sensors}

All perception modules are tested including the two vision-based stereo systems (front and back), the panoramic camera system and all the LIDAR-based systems. The forward stereo vision system is used to locate obstacles, vehicles, pedestrians and lane markings in a short and medium range, while the backward one is used only for obstacle detection in a short range during backup. The panoramic viewing system stitches the three images together to form a single panoramic view of about 180 degrees, in order to locate the leader vehicle even in challenging conditions like winding or hilly roads. The two laser scanners at the extremes of the frontal bull-bar detect obstacles in a short and medium range in front and on the sides of the vehicle. A multibeam laser scanner, installed in front of the vehicle, is used to locate obstacles and the preceding vehicle in short, medium and long ranges. The tilted monobeam laser scanner is used to detect the traversable path, especially when driving off-road: it can locate kerbs, ditches, berms and sidewalks. All units run the same software framework, GOLD (Bertozzi et al., 2008) and the various applications are implemented as separate plugins.

\subsection{Leader-follower navigation}

A leader-follower approach has been used to manage most of the trip: due to the lack of digital maps, the first vehicle defines the route and the second one follows using both a vehicle detector algorithm based on the fusion between the two main technologies mounted onboard (the laserscanners placed inside the front bumper and the panoramic view system placed inside the cabin) and a GPS-based waypoint following. Two situations are possible, depending the leader visibility. If the leader is in line of sight and the follower can locate its shape, then the follower will use the leader position to determine its trajectory; local sensing 
is used to refine its position on the road. In order to increase the field of view of the follower vehicle, the panoramic viewing functionality allows to detect and track the leader vehicle even when approaching a tight curve or a steep hill. On the other hand, when the leader is not visible by the follower (for example it is behind a curve or a third vehicle is in-between), the second vehicle follows the coarse GPS waypoints broadcasted via radio connection by the first vehicle and, again, local sensing is used to refine the vehicle trajectory. The Stop-andGo functionality is also included for speed adjustment with respect to the preceding vehicle.

\subsection{Lane detection}

Navigation in such different environments has to be very precise. Basic perception tasks, such as the detection of painted road markings, are essential components of both intelligent vehicles and advanced road safety systems and are the primary source of information on a rapidly-changing environment.

The vehicles must be able to detect obstacles and lane markings (where available) with high confidence and accuracy. The lane detection system proposed by Felisa and Zani (2010) overcomes the difficulties of identifying the lane boundaries, whose appearance greatly varies depending on illumination, weather and road conditions, using a single camera. The lane detection system has been tested both in leader-follower situations, as help to the path planner and stand alone as a lane keeping system.

\subsection{Obstacle detection}

Obstacle sensing is of great importance since other vehicles, travelling in either directions, or fixed and moving obstacles may be on the vehicle's trajectory. Stereoscopic systems offer great advantages since they allow to reconstruct the $3 \mathrm{D}$ environment providing an easy detection of anything above the road surface. Giving a definition of an obstacle is a non-trivial task; even robust and extensively tested methods that consider everything sticking out of a dominant ground surface as an obstacle can fail in some situations (e.g., very cluttered environments with no clearly visible road area, or lateral slopes). Obstacle detection system during VIAC is performed by clustering the points detected by the disparity engine using a truncated cone shape search area, whose parameters are computed considering the minimum obstacle height, the height of the vehicle and the maximum angle which can be negotiated by the vehicle. Obstacle detection is also performed using laser scanners. The multilayer laser scanner is used as the main sensor, while the other two laser scanners are used to add information in the peripheral areas. The first step is the discarding of echoes refering to ground, using a method already implemented during the DARPA Grand Challenge (Broggi et al., 2006). Pulses are then clustered together to generate obstacles, which are tracked using geometric invariant moments. The two obstacle detection methods have been used together (with a very simple high level fusion) or separately.

\subsection{Ditch and berms detection}

A ditch and berms detection system allows the vehicle to identify the traversable areas. A mono-beam laser scanner is pitched down so that the beam hits the ground in front of the vehicle; the system is designed to be robust and to produce reliable results for both off-road and urban environments. When driving off-road, the vehicle cannot rely on the presence of lane markings and the system provides information about the presence of ditches, bumps, 
berms and obstacles right in front of the vehicle. Navigation in urban environments, on the contrary, requires a precise detection of sidewalks and kerbs, which often delimit the traversable part of the road. Their identification is often a challenging task because of their low height.

\subsection{Path planning}

The VIAC autonomous vehicles control system (Broggi et al., 2010) is capable of using different inputs, such as GPS waypoints, roadways borders and lines, the leader vehicle and obstacles. To fulfill its mission a general-purpose real-time motion planning system, based on the estimation of feasible trajectories on a cost map, was designed and implemented. An optimal trajectory is provided at high frequency in order to cope with the unstable nature of vehicle dynamics. Figure 4 shows the system interface with current navigation data; this image also contains the path planner output image, in which the optimal trajectory is shown.

Figure 4 Systems interface: data and path planner output (see online version for colours)

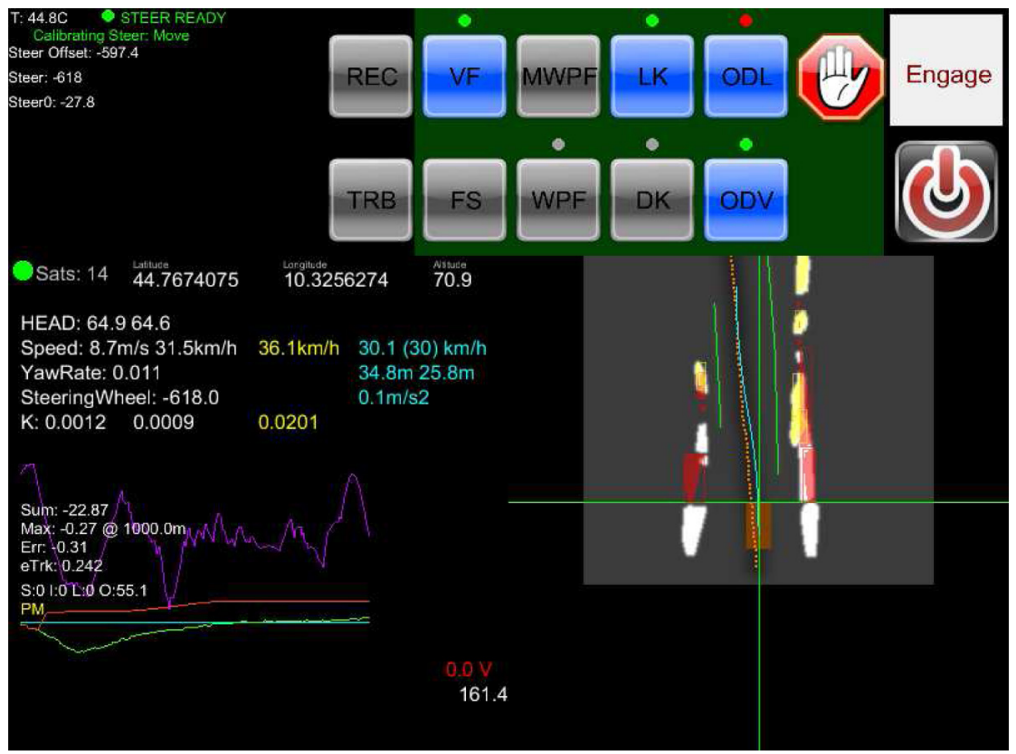

\subsection{Calibration}

During the trip maintenance and check-ups of all vehicles and vision systems were performed regularly, with a few extraordinary interventions like the replacement of the whole battery pack on one of the vans in Moscow and the replacement of two battery chargers near the city of Turpan, China. Calibration checks for the four vehicle-sensing systems (cameras and laser scanners) were also carried out on a regular basis and sometimes recalibration was also performed.

Calibration is a critical task for autonomous driving and specific calibration tools and procedures have been designed by VisLab in order to simplify these operations even in unfriendly conditions. 
The multi-layer laser scanner is calibrated using obstacles in known positions; it is moved until it localises the obstacles in their correct positions. The other laser scanners are calibrated with an auto-calibration software, using the multi-layer laser scanner data as ground truth.

A number of poles are positioned in the area framed by all the sensors and the multi-layer laser scanner is used to compute their position: this information is compared with the measurement provided by the other sensors to compute their extrinsic parameters. This autocalibration procedure provides very low error rates concerning roll, pitch and yaw and aims to support people to reach a precise calibration in a short time.

Stereo cameras' extrinsic parameters are computed using a $6 \times 9 \mathrm{~m}$ calibration grid with a chessboard pattern of fixed size that has been brought along the trip (see Fig. 5). The grid is used to manually map points on image to points in the world. This technique allows to associate a value in metres to each pixel in the image, giving information about distance of objects as shown in Zhang (2000).

Figure 5 Stereo cameras calibration procedure with the chessboard pattern in a car park in Barnaul (Russian Federation): (a) internal view and (b) external view (see online version for colours)

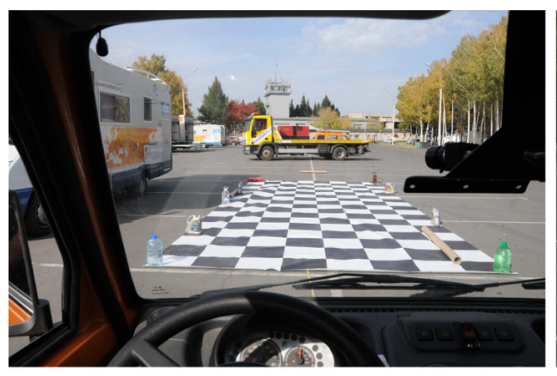

(a)

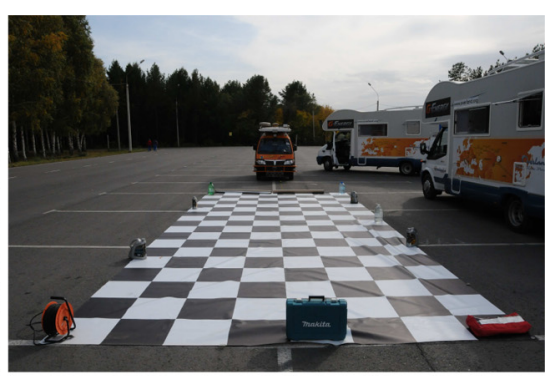

(b)

\subsection{Additional offline processing and testing}

Since a large amount of tests have been planned during the trip, due to lack of time, some tests had to be skipped and delayed till the end of the journey. Therefore all data coming from the whole set of sensors and from the vehicles themselves have been collected, tagged and stored: this allows offline testing, tuning and improvement of another whole set of algorithms which were not used during VIAC, such as terrain mapping and slope estimation, detection of zebra crossings and pedestrians, tunnel and visibility estimation (see Fig. 3c-d).

\section{The route and the results}

\subsection{Route}

The test was carried out across several countries thanks to an extensive human effort: the trip took over three months and covered a total distance of more than 13,000 km.

The vehicles travelled from Italy to China through Slovenia, Croatia, Serbia, Hungary, Ukraine, Russia and Kazakhstan (as depicted in Fig. 6). 
Figure 6 The route covered by VIAC: 13,000 km from Italy to China, as shown on live tracking web page available online during the trip (see online version for colours)

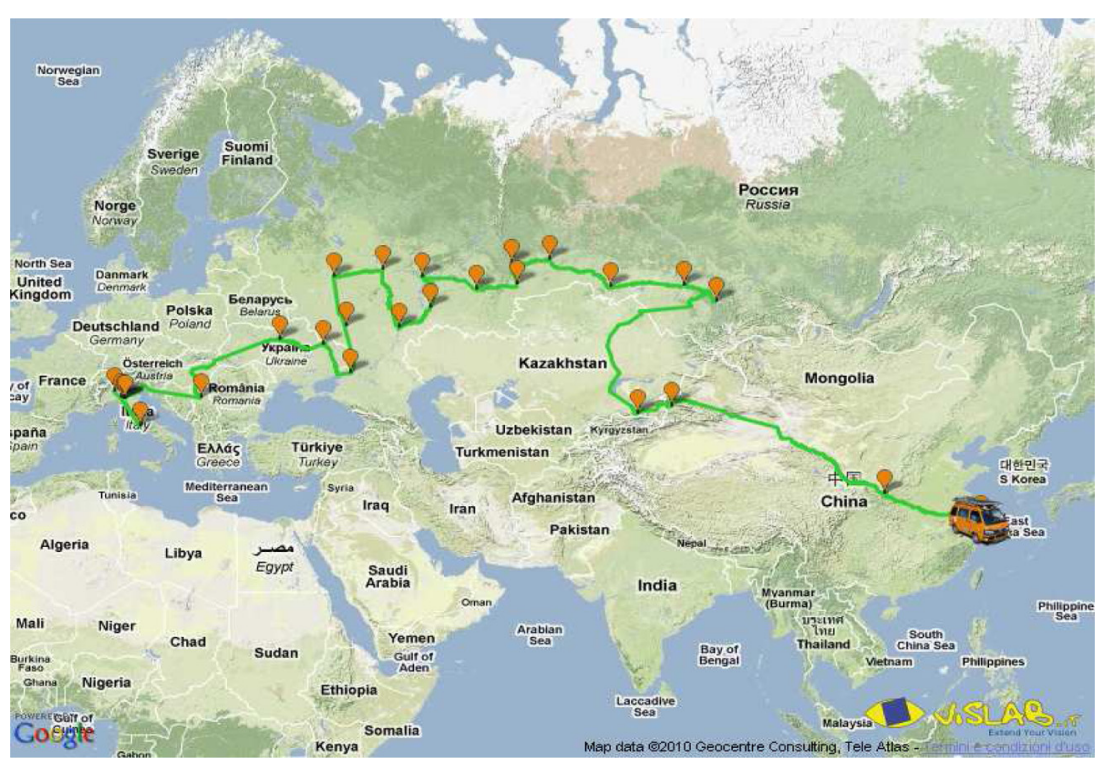

The trip stopped in selected cities along the route where live demos, presentations and press conferences were organised to show case the technologies VisLab developed during the past years.

Once the expedition reached Shanghai, all the vehicles were loaded on the convoy support trucks and transported back to Italy.

\subsection{Scenarios}

Throughout the journey the expedition met a plurality of completely different scenarios: urban and off-road, highways, mountains and plateaus. In Europe and in the first part of the Russian Federation the convoy travelled for many kilometres on highways and drove into the heavy urban traffic of many great eastern Europe cities including Belgrade, Budapest, Kiev, Moscow, then it went across Siberia, the flat steppes of Kazakhstan, up the Tien Shan Mountains and finally the vehicles reached Shanghai.

The weather also changed dramatically, from the hot summer of Ukraine to the cold September in Russia, the pouring rain of China and snow on the mountain passes. It was impossible to predict all the environments and situations that the vehicles had to face, but this was one of the main purposes of the test: to design algorithms as robust as possible, evaluate their performance and improve them afterwards using the data collected in the most critical scenarios.

\subsection{Elevation map}

Although most of the journey took place on fairly flat countries, in China the convoy had to pass through the Tien Shan Mountains $(2100 \mathrm{~m}$ ) and the Lanquan pass at almost $3000 \mathrm{~m}$, the highest point reached during the whole trip. The elevation map in Figure 7 shows the height provided by the GPS sensors recorded during the whole journey. 
Figure 7 Elevation map: the most significant altitudes recorded (see online version for colours)

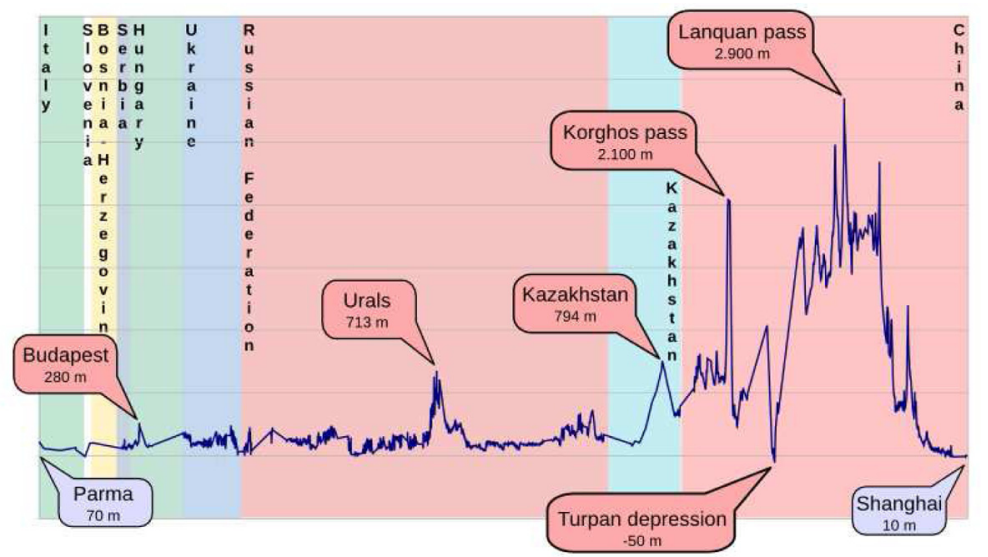

In these scenarios road conditions were bad, with a lot of dust, large holes and off-road tracks and the driving speed dropped due to the uphill driving (See Fig. 8). Moreover, battery life decreased considerably and performing autonomous runs became more challenging.

Figure 8 Off-road driving on a Chinese road under construction (see online version for colours)

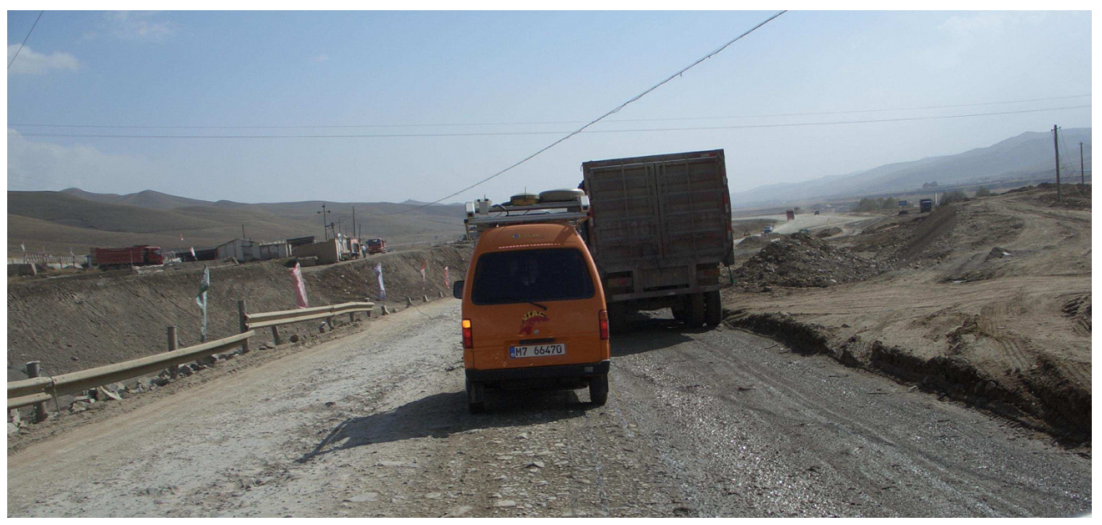

\subsection{Temperatures}

The chart in Figure 9 shows the temperatures collected throughout the entire expedition. Red, green and blue lines show each day maximum, average and minimum temperatures respectively. Data were acquired by a sensor inside the GPS antenna which was positioned on the vehicle rooftop, so measured values were biased: the highest temperature was increased by sun exposure while the minimum was altered by sensor's motherboard heat.

Nonetheless data trend remained interesting, with a maximum temperature of about $50^{\circ} \mathrm{C}$ recorded in Europe during summer months and a minimum one of nearly $0^{\circ} \mathrm{C}$ registered in Siberia where the expedition faced cold weather with a short snowfall as well. 
Figure 9 Temperatures recorded during the trip (see online version for colours)

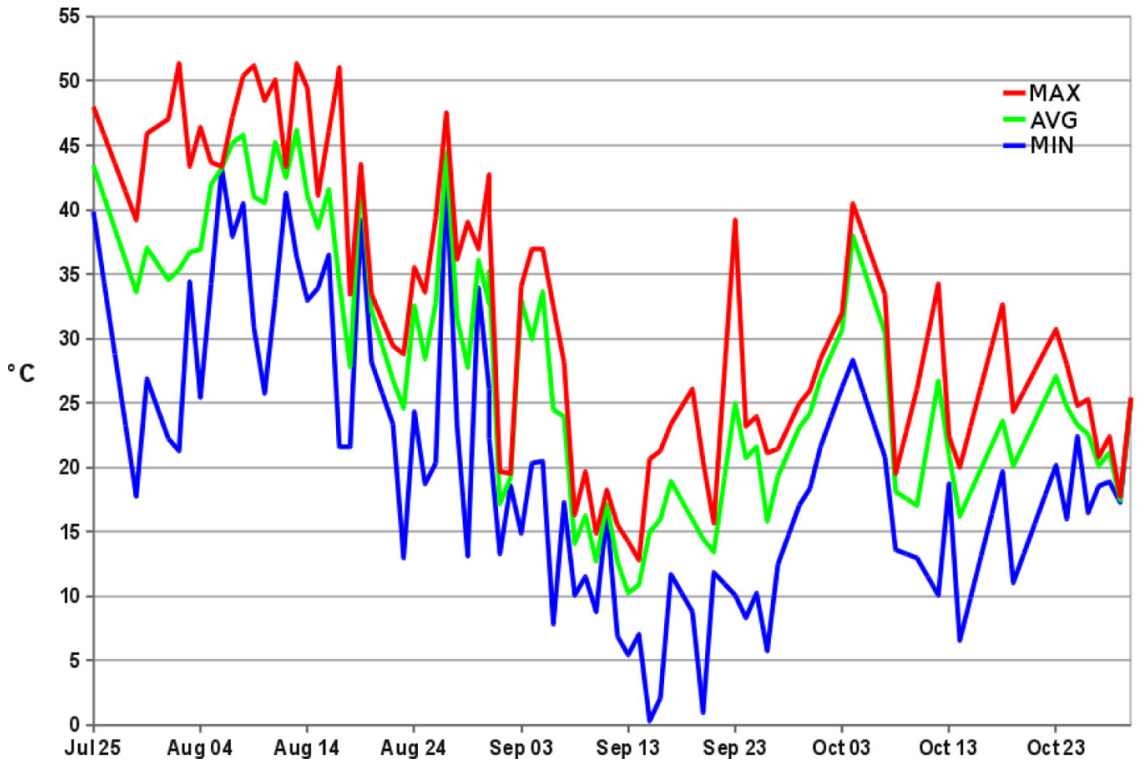

\subsection{Test patterns}

According to the leader-following approach, two autonomous vehicles were moving at the same time during the trip but with different behaviours, although all the vehicles were exactly identical. Every day the vehicles were able to perform from two to four autonomous runs.

Early in the morning, the batteries of all four vehicles were fully charged and the first pair of vehicles was prepared for the first autonomous run of the day while the other two vehicles were loaded on the trucks. When their batteries were exhausted, the convoy stopped to swap the vehicles.

This was a quite complex operation requiring a quite large area to perform all the operations which was not easy to spot both in crowded areas and in remote locations. For this reason, sometimes the vehicles were swapped in rest areas along highways even if the batteries were not completely discharged thus reducing the already quite limited vehicle's autonomy. At each swap the following instrumentation had to be moved from one vehicle to the next:

- The satellite tracking antenna which was positioned on the rooftop of the leader vehicle and the transreceiver terminal inside the same vehicle. They were moved from a leading vehicle to the other.

- The webcam and the PC which were used to broadcast live images directly from the journey.

- Two laptop PCs which were used to connect with the vehicle systems (both leader and follower).

- The $\mathrm{CO}_{2}$ sensors and the smartphone placed respectively on the rooftop and inside the cabin of one of the vehicles (leader or follower). 
During the second autonomous run the first pair of vehicles started recharging on the tow truck; once the batteries of the second pair of vehicles were exhausted the convoy had to stop and wait for the complete charge of the batteries. The team, after connecting the other two vehicles to the generator, usually spent this time having lunch and doing maintenance on the vehicles.

In the afternoon another autonomous run was performed while the second pair completed the battery charging, then the vehicles were swapped again and the fourth run started; at the end of this last run the convoy stopped and the vehicle batteries were connected to the power generator for the whole night to allow equalisation and full recharge.

\subsection{The last run in Shanghai}

The last run of the expedition was done accessing Shanghai from one of its main entrances, the great Nanpu Bridge. The vehicles made the last part of the trip followed by several groups of journalists while facing Shanghai downtown heavy traffic conditions. This run was done in fully autonomous mode for both the leader and the follower and since it was quite long, a swap was also performed. The vehicles also drove around the city in leaderfollower configuration and reached one of Shanghai's most popular sites, Waibaidu Bridge surrounding area with a great view over the Huangpu River looking out to the futuristic buildings of the Pudong quarter (see Fig. 10(a)).

Figure 10 The vans in Shanghai's downtown renowned Bund area (a) and inside the Expo, displayed at the Belgium-EU pavilion (b), during the public parade (c), the Expo parade GPS path in green (d) (see online version for colours)

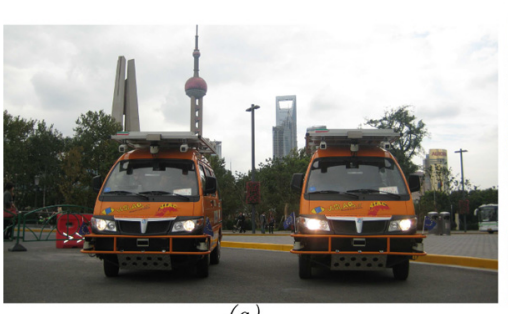

(a)

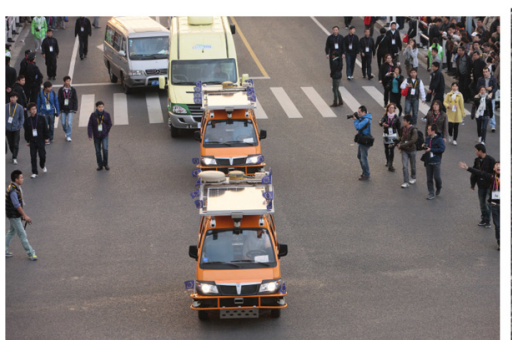

(c)

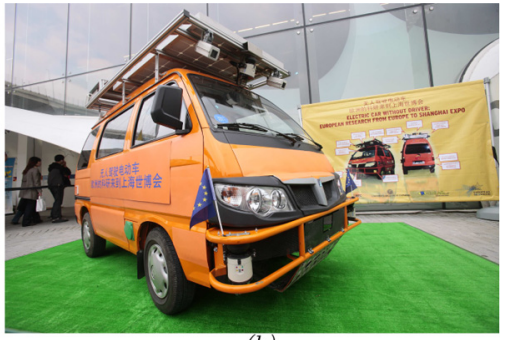

(b)

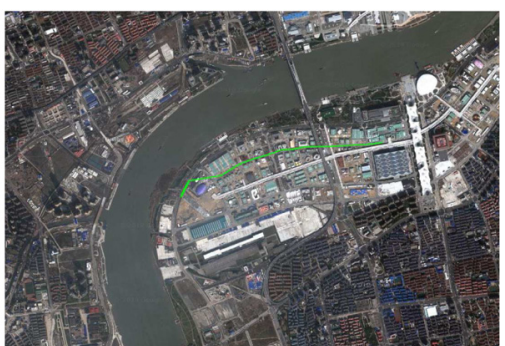

(d)

VIAC had is official conclusion on October the 28th 2010 at the EU pavilion inside Shanghai's 2010 World Expo. A conference was held at the presence of the European Community representatives and one of the autonomous vans was shown outside the building (Fig. 10).

The final part of the event was a demonstration of the leader-following system with two automatic vehicles which took part in a choreographic parade with carnival floats passing through the exposition pavillions, as shown in Figures 10(c)-(d). 


\subsection{Statistics}

The collected data refer to the 61 effective days of autonomous driving on an overall 90 days journey. About 16 days were spent giving demos and press conferences in a number of towns along the route. 191 runs for a total of 214 hours in autonomous mode were completed. $8244 \mathrm{~km}$ were covered in autonomous mode, at an average speed of $38.4 \mathrm{~km} / \mathrm{h}$ and a maximum speed of $70.9 \mathrm{~km} / \mathrm{h}$. A detailed speed profile is reported in Figure 11. The maximum distance covered in a single day was of $273 \mathrm{~km}$ and the maximum amount of time spent in a day driving in autonomous mode was of $6 \mathrm{~h}: 26 \mathrm{~min}$.

In Figure 12(a) statistical path-planner error graph is shown. This graph is obtained using the trajectory followed by the leader vehicle as ground-truth. The histogram represents

Figure 11 Overall profile of the electric vehicles speed during the journey

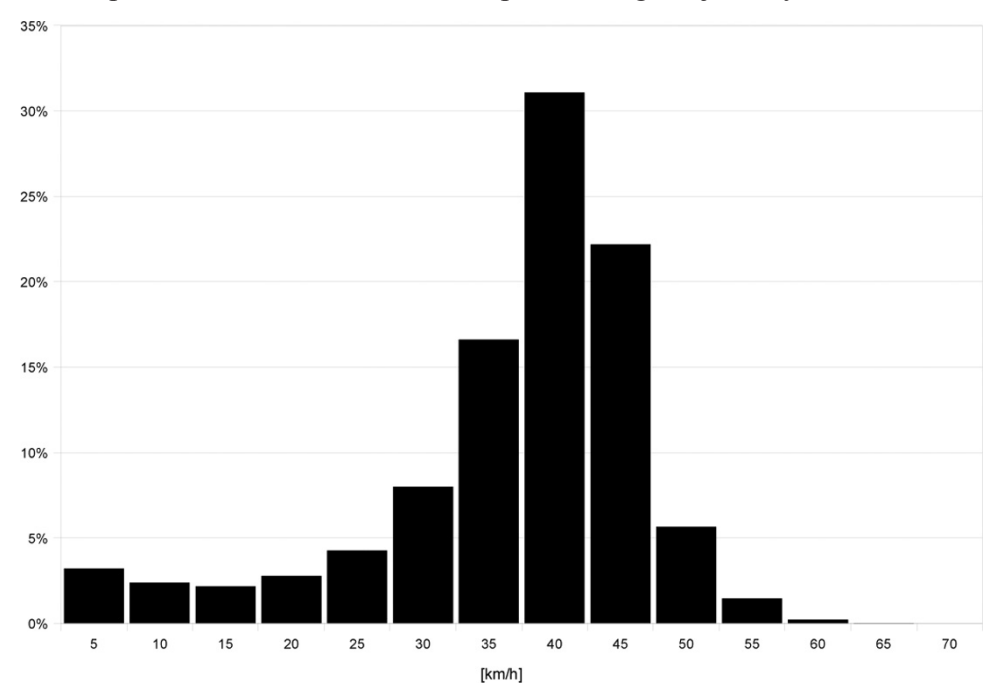

Figure 12 Histograms of lateral cross-track errors in the autonomous driving configuration (Leader Following)

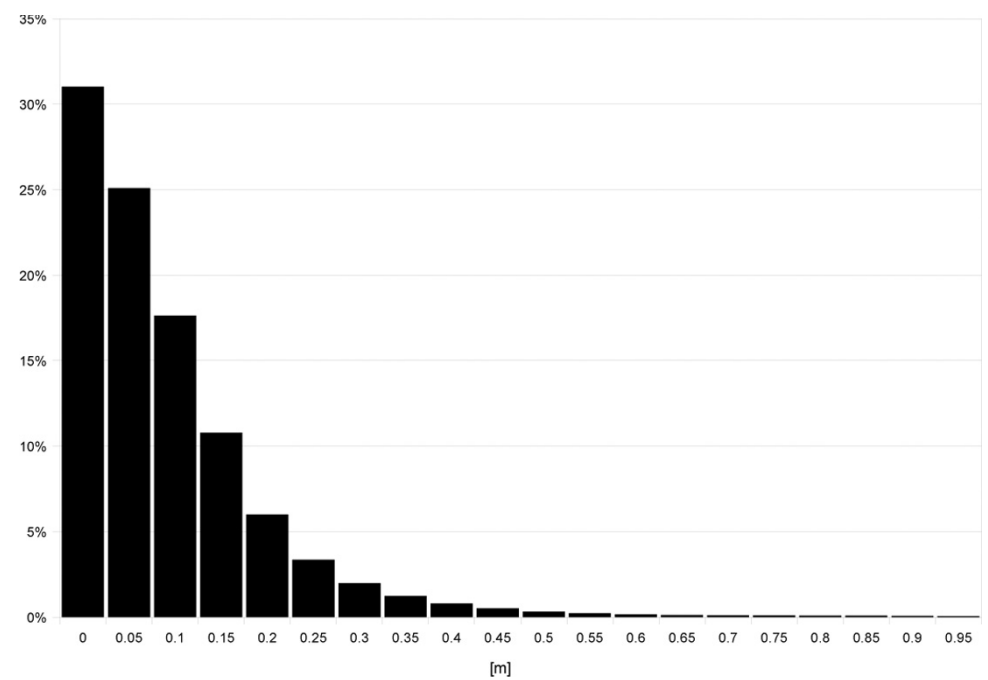


the distribution of the differences between the path of the leader and follower. Differences between the two paths are due to the fact that the following vehicle used the leader's trajectory as an indicative path only and merged it with data provided by the other vision systems to generate its own optimal trajectory.

\section{Conclusions}

The VIAC covered a distance of $13,000 \mathrm{~km}$ through different countries from Italy to China in autonomous driving mode and was the longest and most extreme test of autonomous driving so far. During the whole journey the four vehicles had to face many kinds of environmental conditions, road scenarios and weather situations.

The journey had a great scientific and media impact and it is now considered a milestone in autonomous driving research.

The main difference between VIAC and other autonomous driving tests, demo or competitions, like DARPA Grand Challenge and Urban Challenge, was that VIAC got across normal road in normal conditions and no assumptions could be done about traffic, scenarios and respect of traffic rules.

Unlike in previous tests, no maps were available and it was not possible to plan the route or to predict complex situations or specific scenarios.

The key reason for this journey was not a demo of the systems, but a long test to improve and check systems performance of the driveless technology developed by VisLab in the last 15 years.

Some preliminary results were presented in this paper; anyway a large amount of data has been collected during the whole journey (about 50 terabyte), which are still being processed. It is estimated that their unique set of data will bring an enourmous value to our research and to our partners, at least for the next 2-3 years.

\section{Acknowledgements}

The work described in this paper has been developed in the framework of the Open intelligent systems for Future Autonomous Vehicles (OFAV) Project funded by the European Research Council (ERC) within an Advanced Investigators Grant.

\section{References}

Bertozzi, M., Bombini, L., Broggi, A., Cerri, P., Grisleri, P. and Zani, P. (2008) 'GOLD: a complete framework for developing artificial vision applications for intelligent vehicles', IEEE Intelligent Systems, Vol. 23, No. 1, pp.69-71.

Bombini, L., Cattani, S., Cerri, P., Fedriga, R.I., Felisa, M. and Porta, P.P. (2009) 'Test bed for unified perception \& decision architecture', in Procs., 13th Int., Forum on Advanced Microsystems for Automotive Applications, Berlin, Germany, pp.287-298.

Braid, D., Broggi, A. and Schmiedel, G. (2006a) 'The TerraMax autonomous vehicle', Journal of Field Robotics, Vol. 23, No. 9, pp.693-708.

Braid, D., Broggi, A. and Schmiedel, G. (2006b) 'The TerraMax autonomous vehicle concludes the 2005 DARPA grand challenge', in Procs. IEEE Intelligent Vehicles Symposium 2006, Tokyo, Japan, pp.534-539.

Broggi, A., Bertozzi, M. and Fascioli, A. (1999) 'ARGO and the MilleMiglia in Automatico Tour', IEEE Intelligent Systems, Vol. 14, No. 1, pp.55-64. 
Broggi, A., Cattani, S., Porta, P.P. and Zani, P. (2006) 'A Laserscanner-Vision fusion system implemented on the terramax autonomous vehicle', in Procs. IEEE/RSJ Intl. Conf. on Intelligent Robots and Systems, Beijing, China, pp.111-116.

Broggi, A., Medici, P., Cardarelli, E., Cerri, P., Giacomazzo, A. and Finardi, N. (2010) 'Development of the control system for the VisLab intercontinental autonomous challenge', in Procs. IEEE Intelligent Transportation Systems 2010, pp.635-640, ISSN: 2153-0009.

Chen, Y-L., Sundareswaran, V., Anderson, C., Broggi, A., Grisleri, P., Porta, P.P., Zani, P. and Beck, J. (2008) 'TerraMax: team oshkosh urban robot', Journal of Field Robotics, Vol. 25, No. 10, pp.841-860.

Chen, Y-L., Sundareswaran, V., Anderson, C., Broggi, A., Grisleri, P., Porta, P.P., Zani, P. and Beck, J. (2009) 'TerraMax: team oshkosh urban robot', in Buehler, M., Iagnemma, K. and Singh, S. (Eds.): The DARPA Urban Challenge, Autonomous Vehicles in City Traffic, Springer Tracts in Advanced Robotics, Springer-Verlag Berlin Heidelberg, pp.595-622, ISBN: 978-3-642-03990-4.

Felisa, M. and Zani, P. (2010) 'Robust monocular lane detection in urban environments', in Procs. IEEE Intelligent Vehicles Symposium 2010, San Diego, CA, USA, pp.591-596.

Grisleri, P. and Fedriga, I. (2010) 'The BRAiVE platform', in Procs. 7th IFAC Symposium on Intelligent Autonomous Vehicles, Lecce, Italy.

IBM (2010) The Greenhaviour Project at the VIAC Challenge, Available at http://www.greenhaviour. org

Ozguner, U., Redmill, K A. and Broggi, A. (2004) 'Team TerraMax and the DARPA grand challenge: a general overview', in Procs. IEEE Intelligent Vehicles Symposium 2004, Parma, Italy, pp.232-237.

van Dijke, J.P. (2009) 'CityMobil, advanced transport for the urban environment', 988th Annual Meeting of Transportation Research Board, TRB - Transportation Research Board, Washington DC.

Venturi (2010) Venturi Global Challenges, Available at http://www.venturi.fr/Venturi-globalchallenges 2

Zhang, Z. (2000) 'A flexible new technique for camera calibration', IEEE Transactions on Pattern Analysis and Machine Intelligence, Vol. 22, pp.1330-1334. 